\title{
Synthesis and Characterization of Highly Efficient Nickel Nanocatalysts and Their Use in Degradation of Organic Dyes
}

\author{
Nazar Hussain Kalwar, ${ }^{1}$ Sirajuddin, ${ }^{1}$ Razium Ali Soomro, ${ }^{1}$ Syed Tufail Hussain Sherazi, ${ }^{1}$ \\ Keith Richard Hallam, ${ }^{2}$ and Abdul Rauf Khaskheli ${ }^{1,3}$ \\ ${ }^{1}$ National Centre of Excellence in Analytical Chemistry, University of Sindh, Jamshoro 76080, Pakistan \\ ${ }^{2}$ Interface Analysis Centre, School of Physics, University of Bristol, Bristol BS8 1TL, UK \\ ${ }^{3}$ Department of Pharmacy, Shaheed Mohtarma Benazir Bhutto Medical University, Larkana 77150, Pakistan
}

Correspondence should be addressed to Razium Ali Soomro; raziumsoomro@gmail.com

Received 10 October 2013; Revised 4 January 2014; Accepted 9 January 2014; Published 27 February 2014

Academic Editor: Sepperumal Murugesan

Copyright (C) 2014 Nazar Hussain Kalwar et al. This is an open access article distributed under the Creative Commons Attribution License, which permits unrestricted use, distribution, and reproduction in any medium, provided the original work is properly cited.

The present study describes the synthesis of highly active and ordered structures of nickel nanocatalysts by a facile, green, and economically viable approach. The study reveals efficient catalytic activity for the degradation of a number of toxic organic dyes, such as eosin-B (EB), rose bengal (RB), eriochrome black-T (ECBT), and methylene blue (MB). The stable ordered nickel nanostructure (Ni NSs) arrays were prepared via a modified hydrazine reduction route with unique and controlled morphologies in a lyotropic liquid crystalline medium using a nonionic surfactant (Triton X-100). Characterization and optimization studies for the fabricated Ni NSs involving their surface binding interactions, size, and morphologies were carried out using UV-Vis spectroscopy, Fourier transform infrared (FTIR) spectroscopy, X-ray diffraction (XRD), and scanning electron microscopy (SEM).

\section{Introduction}

The widespread interest in the synthesis of metal nanomaterials has largely been driven by their potential applications in various fields ranging from sensors and optical switches to catalysis [1]. Similarly, the control over pollution, especially contamination of the aquatic environment due to industrial effluents, has become an increasingly important and complex issue of present day research concern. The existence of industrially important dyes in drinking water may pose a serious threat to human health. Since there are both environmental and health concerns in this particular issue, there is a need to introduce efficient and inexpensive protocols for the treatment of waters and effluents [2]. A variety of methodologies have been developed for investigation of environmental contaminants which exploit the reactivity of target molecules with suitable reagents alone or in the presence of catalysts, ultimately resulting in degradation, removal, or safe disposal of environmental and aquatic pollutants $[3,4]$. Some of the water treatment methods include, adsorption method $[5,6]$, biological discoloration [7], and advanced oxidation processes such as the photo-fenton reaction [8], photocatalytic degradation by UV irradiation [9], visible light [10], and microwave discharge lamps $[11,12]$ are well regarded for the removal or degradation of dyes from wastewaters.

However, each of these technologies exhibits their own limitations. For example, dyes cannot always be completely eliminated by biological methods because most dyes are recalcitrant molecules; the pollutants are not degraded by adsorption but are just transferred from one phase to the other and advanced oxidation processes are limited due to high energy requirements and expensive operating costs [13, 14]. The UV-induced degradation of industrially important azo dyes such as methyl orange (MO) and methyl red (MR) has been carried out by employing different catalysts. The reduction for $\mathrm{MO}$ was $38 \%$ for $\mathrm{nh}-\mathrm{ZnO}, 89 \%$ for 
$\mathrm{ZnO}$ Aldrich, and 95\% for $\mathrm{h}-\mathrm{ZnO}$. Further, the percentage reduction for $\mathrm{MR}$ was $54 \%$ for $\mathrm{nh}-\mathrm{ZnO}, 93 \%$ for $\mathrm{ZnO}$ Aldrich, and $100 \%$ for h-ZnO; all these were obtained after 120 minutes [15]. Degradation and decolorization of acid black 26 dye have also been studied through a heterogeneous catalytic approach. It was established that, in the presence of $\mathrm{TiO}_{2}$ particles alone, acid black 26 (dye: $0.071 \mathrm{mM}, \mathrm{H}_{2} \mathrm{O}_{2}$ : $0.023 \mathrm{mM}$ ) was degraded to a greater extent than $\mathrm{TiO}_{2}$ coated sackcloth fiber after $60 \mathrm{~min}$. They observed that it took $3 \mathrm{~h}$ to effectively decompose all dye molecules in solution using $\mathrm{TiO}_{2}$-coated sackcloth fiber [16]. In another report, Ag-HDx-g-PAM nanocomposite was used for the reduction of pollutants like aromatic nitro compounds and phenosafranine $\left(\mathrm{PS}^{+}\right)$dye. It was seen that, after the addition of nanocomposites to the mixture of reducing agent and dye $\left(0.01 \mathrm{M} \mathrm{NaBH}_{4}\right.$ and $\left.0.05 \mathrm{mM} \mathrm{PS}^{+}\right)$, the solution changed from light pink to yellowish brown, due to reduction of the dye. They observed complete reduction of the dye in $30 \mathrm{~min}$ after addition of $0.0065 \mathrm{wt} \%$ of Ag-HDx-g-PAM nanocomposite [17]. The role of $\mathrm{MnFe}_{2} \mathrm{O}_{4}$ mesoporous composites in the degradation of methyl orange (MO) dye was investigated. The MO solution was completely degraded by $\mathrm{MnFe}_{2} \mathrm{O}_{4}$ mesoporous composites after $420 \mathrm{~min}$, using $20 \mathrm{mg}$ of the catalyst [18].

In a previous study, it has been reported that the catalytic reduction of dyes was carried out by using gelatin stabilized gold nanoparticles (GNPs), where the entire reduction process was completed in $150 \mathrm{~s}$ at room temperature with only $0.12 \mathrm{mg}$ GNPs [19]. In another report, it has been described that nanoscale nickel particles (Ni NPs) are highly efficient catalysts for reduction of congo red (CR, $20 \mu \mathrm{M})$ dye from aqueous medium, where $100 \%$ reduction/degradation of dye was observed in a very short time $(50 \mathrm{~s})$ by the addition of only $0.2 \mathrm{mg} \mathrm{Ni} \mathrm{NPs}$ in the presence of reducing agent (100 mM NaBH4) [20]. Hence, the literature demonstrated that nanostructured $\mathrm{Ni}$ catalysts are highly efficient and important alternative to the already available catalysts while being able to be easily recovered for use through several cycles without loss of catalytic efficiency. Further studies have been carried out giving much attention to probing control over the morphology and growth patterning of Ni NSs and the effect of different parameters on catalytic efficiency in terms of reaction kinetics. However, the prime objective of this present study is to explore the synthesis of Ni NSs and their catalytic activity for the degradation of environmentally toxic and hazardous organic dyes.

\section{Experimental}

2.1. Materials Used during Synthesis of TX-100 Stabilized Ni NSs. The nickel (II) chloride hexahydrate (97\%), hydrazine monohydrate (99\%), Triton X-100 (100\%, the stabilizing agent), pellets of sodium hydroxide (99\%), hydrochloric acid (37\%), and sodium borohydride (98\%) were all purchased from E. Merck. Due to possible oxidation of reagents, fresh solutions of chosen concentrations were prepared in deionized water for each experiment. All these reagents were analytical grade and were used without additional purification.

2.2. Instruments Used during Characterization of TX-100 Stabilized Ni NSs. The optimization studies of various parameters were monitored by recording UV-Vis spectra on a Shimadzu UV-160 digital spectrophotometer (Kyoto, Japan) with a $1 \mathrm{~cm}$ quartz cuvette. A Nicolet 5700 FT-IR spectrophotometer was used to record FTIR spectra. A Jeol JSM 6380A SEM was used to image the nanostructures. A Bruker D8 ADVANCE diffractometer was used to record X-ray diffraction patterns from $20^{\circ}$ to $80^{\circ}$ using $\mathrm{CuK} \alpha$ radiation.

2.3. Synthesis Procedure of TX-100 Stabilized Ni NSs. A typical experiment was performed by mixing $\mathrm{NiCl}_{2} \cdot 6 \mathrm{H}_{2} \mathrm{O}(0.5 \mathrm{~mL}$, $0.033 \mathrm{M}), \mathrm{NaOH}(0.3 \mathrm{~mL}, 0.1 \mathrm{M}), \mathrm{N}_{2} \mathrm{H}_{4} \cdot \mathrm{H}_{2} \mathrm{O}(1.0 \mathrm{~mL}, 0.2 \mathrm{M})$, and Triton X-100 $(0.5 \mathrm{~mL}, 0.5 \mathrm{M})$ at room temperature; the solution was then diluted to $10 \mathrm{~mL}$ with deionized water. As the reaction proceeded, after a few minutes a light blue color appeared as sufficient quantities of reducing agent were added that gradually became deeper with increase in the quantity of reducing agent.

2.4. Catalytic Test of TX-100 Stabilized Ni NSs. Investigation of the catalytic efficiency of newly synthesized Ni NSs for degradation of a number of organic dyes has been carried out; fresh samples of Ni NSs were prepared and deposited on glass cover slips for each experiment. For efficient adhesion of $\mathrm{Ni}$ NSs onto glass surfaces, the deposited catalyst was thermally treated on the cleaned surface of an electric hotplate at $150^{\circ} \mathrm{C}$ for $10 \mathrm{~min}$. The supported catalyst of suitable quantity was placed in a quartz cell and then inside a cuvette in opposition to the light beam to monitor in situ the course of reaction by UV-Vis spectroscopy.

A typical catalytic test of Ni NSs for degradation of organic dyes was carried out under normal laboratory conditions. Organic dyes such as eosin-B (EB), rose bengal (RB), eriochrome black-T (ECBT), and methylene blue (MB) were selected as target compounds to check the efficiency of the catalyst. All experiments were performed in an aqueous medium using $20 \mu \mathrm{M}$ concentration of the respective dyes, $0.01 \mathrm{M} \mathrm{NaBH}_{4}$ (reducing agent), and $0.1 \mathrm{mg}$ quantity of $\mathrm{Ni}$ NSs (catalyst). A similar experiment was also performed for a mixture of all four dyes with $20 \mu \mathrm{M}$ concentration of each dye, $0.01 \mathrm{M} \mathrm{NaBH}_{4}$ (reducing agent), and $0.1 \mathrm{mg}$ quantity of Ni NSs (catalyst). UV-Vis spectra were recorded every $10 \mathrm{~s}$ during the course of reaction.

We observed that in the absence of Ni NSs, there was no reduction/degradation of $\mathrm{MB}$ and $\mathrm{RB}$ dyes and $\mathrm{EB}$ and ECBT were degraded only to a small extent, whereas all dyes were completely degraded in a very short time when treated with $\mathrm{NaBH}_{4}$ in the presence of $0.1 \mathrm{mg} \mathrm{Ni} \mathrm{NSs}$; complete degradation of RB dye was observed within 20 s, EB degraded in $30 \mathrm{~s}$, other dyes such as ECBT and MB were degraded within $40 \mathrm{~s}$, and the mixture of all four dyes was degraded in $60 \mathrm{~s}$.

The Ni NSs were reused several times to confirm the retained catalytic efficiency. The experiments were carried 
out to observe complete reduction/degradation of $20 \mu \mathrm{M}$ concentrations of each dye in very short times (i.e., 20-60 s).

\section{Results and Discussion}

Formations of mixed nickel nanostructures (Ni NSs), sheets/ foils, cubes, and spheres with controllable morphologies have been described using a nonionic surfactant (Triton X-100) as a stabilizing agent under normal laboratory conditions in an aqueous solution. For nanosized Ni NSs prepared using hydrazine as the reducing agent, the mechanism for reduction is similar to that of our previous report and further references therein [21].

Consider

$$
2 \mathrm{Ni}^{2+}+\mathrm{N}_{2} \mathrm{H}_{4}+4 \mathrm{OH}^{-} \longrightarrow 2 \mathrm{Ni}+\mathrm{N}_{2} \uparrow+4 \mathrm{H}_{2} \mathrm{O}
$$

\subsection{Characterization of TX-100 Stabilized Ni NSs}

3.1.1. UV-Vis Spectroscopy of TX-100 Stabilized Ni NSs. The nanometer size regime of newly synthesized nickel structures was spectrophotometrically monitored; these exhibited a characteristic absorption profile in the range 350-400 nm (Figure 1), in close agreement to previously reported work [21].

Depending on the nature of sample/solution, Ni particles generated an absorption edge in the UV-Vis spectral range $374-422 \mathrm{~nm}$, corresponding to the surface plasmon resonance (SPR) band of nanosized Ni particles after reduction generated by $\mathrm{Ni}$ (II) ions [22, 23]. Optimization studies for reducing agent concentration, surfactant concentration, and $\mathrm{pH}$ were carried out to check their effect on the peak shape to further help in the estimation of structure/morphology and size of nanoscale metal particles. The photoevolution of $\mathrm{Ni}$ particles suggests that the change in nature and profile of UV-Vis spectra is due to complete reduction of $\mathrm{Ni}$ (II) ions in surfactant solutions. The stability of Ni NPs was studied by aging solutions for many days. It was observed that surfactant molecules impart stability to the colloidal dispersions/clusters and also control morphology and size of the crowded nanoparticles/structures $[17,24]$.

The appearance of the faint blue color of the sample solution and progression of the spectral profile resulted in a blue shift in $\lambda_{\max }$ from $391 \mathrm{~nm}$ to $366 \mathrm{~nm}$ with gradual increase in absorbance by increasing the reducing agent. This was observed by changing the concentration of the reducing agent gradually as $0.2 \mathrm{~mL}, 0.4 \mathrm{~mL}, 0.6 \mathrm{~mL}, 0.8 \mathrm{~mL}$, and $1.0 \mathrm{~mL}$ of $0.2 \mathrm{M}$ hydrazine hydrate solution $\left(\mathrm{N}_{2} \mathrm{H}_{4} \cdot \mathrm{H}_{2} \mathrm{O}\right)$; the corresponding UV-Vis spectra recorded for these samples are presented in Figure 1(a). The development of the spectral profile of TX-100 stabilized Ni NSs recorded after increase in concentration of surfactant (stabilizing/capping) is absolutely different in the present scenario from that of previous results, as the present study reveals that gradual increases in concentration of stabilizing/capping agent generated a red shift in $\lambda_{\max }$ from $382 \mathrm{~nm}$ to $393 \mathrm{~nm}$, as shown in Figure 1(b), due to the creation of a larger micelle network formed by surfactant molecules in the adopted wet chemical method.
These UV-Vis spectral results were obtained by stepwise increase in concentration as $0.25 \mathrm{~mL}, 0.5 \mathrm{~mL}, 0.75 \mathrm{~mL}$, and $1.0 \mathrm{~mL}$ of Triton X-100 (0.5 M) solutions, Figure $1(\mathrm{~b})$. The formation of larger micelle networks at high concentrations might be due to the presence of attractive intermicellar interactions which lead to enhanced micellar growth and potential agglomeration of micelles to larger spherical particles [25].

In principle, for practical increases in surfactant concentrations, the red shift in $\lambda_{\max }$ (from $382 \mathrm{~nm}$ to $393 \mathrm{~nm}$ ) is linear with concentration and can be credited to an increase in micellar size. The study shows that UV-Vis spectra recorded at different $\mathrm{pH}$ values of the samples/solutions exhibited distinctive absorption maxima, as shown in Figure 1(c), that explore the formation of nanoscale nickel structures with different size and shapes.

The selection of samples at different $\mathrm{pH}$ was carried out to see the effect of $\mathrm{pH}$ on size and shapes of Ni NSs; the lowest observed $\lambda_{\max }$ value was from $\mathrm{pH}$ 9.4. Moreover, due to the formation of highly aggregated nanoparticles (precipitates), there was no well resolved peak resulting from the solution above $\mathrm{pH} 9.4$; hence, it is estimated that the optimal $\mathrm{pH}$ was 9.4. It is also understood that the surfactant molecules cover the surface of nanoscale nickel particles giving them stable size and shape, shown schematically in Figure 2.

Further studies were carried out using FTIR spectroscopy, SEM, and XRD for structural elucidation and rationalization of interaction of surfactant molecules with particles to confirm size and morphologies of the TX-100 stabilized Ni NSs.

3.1.2. Fourier Transform Infrared (FTIR) Spectroscopy. FTIR spectra were recorded to examine the interaction of TX100 molecules with the surfaces of Ni NSs, as presented in Figure 3, which shows that extended complexity and functionality of the nanoscale systems are predictable from efficient linkages employed by the $\mathrm{OH}$ group of TX-100 molecules and $\mathrm{Ni}$ particles obtained in lyotropic liquid crystalline medium.

This study showed that a very strong absorption band at $2870 \mathrm{~cm}^{-1}$, in connection with a weak signal at $3480 \mathrm{~cm}^{-1}$, appeared in the FTIR spectra of absolute TX-100 (standard/pure) due to the presence of free $\mathrm{OH}$ groups in the surfactant molecules, whereas TX-100 derived Ni nanostructures with nanosheet and nanosphere morphologies initiated the appearance of a weak but broad signal at $3260 \mathrm{~cm}^{-1}$ at the optimized $\mathrm{pH}$ of 9.4 . Basic $\mathrm{pH}$ resulted in a band at $3260 \mathrm{~cm}^{-1}$ due to high concentrations of $\mathrm{OH}$ moieties in the products. The additional signals in the FTIR spectra of the Ni NSs obtained at pH 7.3 and pH 4.2 (as shown in Figure 3) were due to interactions of zwitterionic moieties with particle surfaces at nearly neutral $\mathrm{pH}$ while those obtained from acidic $\mathrm{pH}$ were expected to be due to strong linkages imparted from amino groups with particle surfaces.

3.1.3. Scanning Electron Microscopy (SEM) of TX-100 Stabilized Ni NSs. Fabrication of nanoscale materials with ordered and controlled structures has been given intensive attention due to their fascinating properties, because the performance 


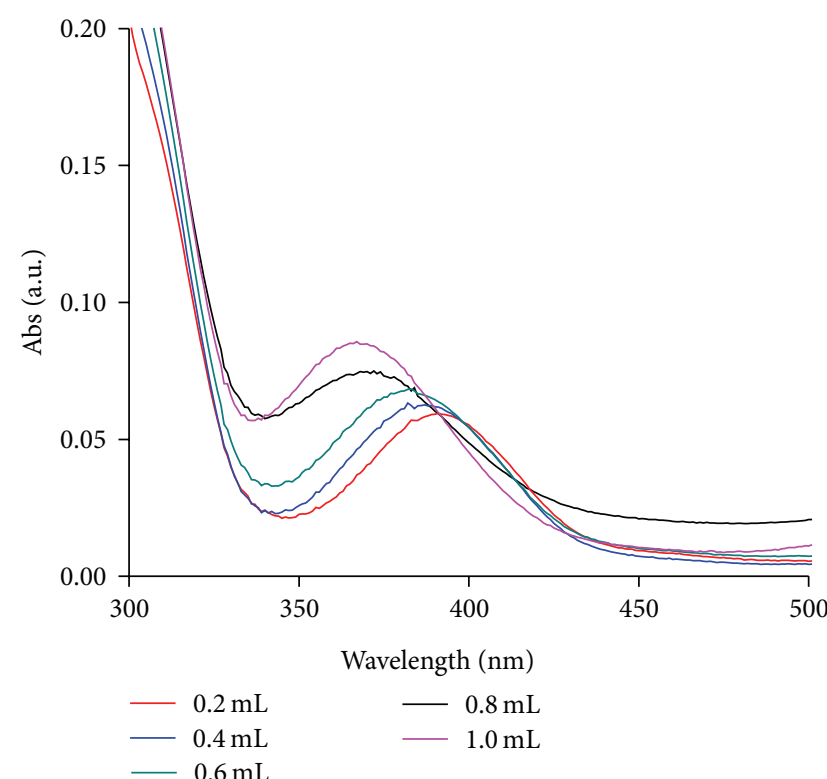

(a)

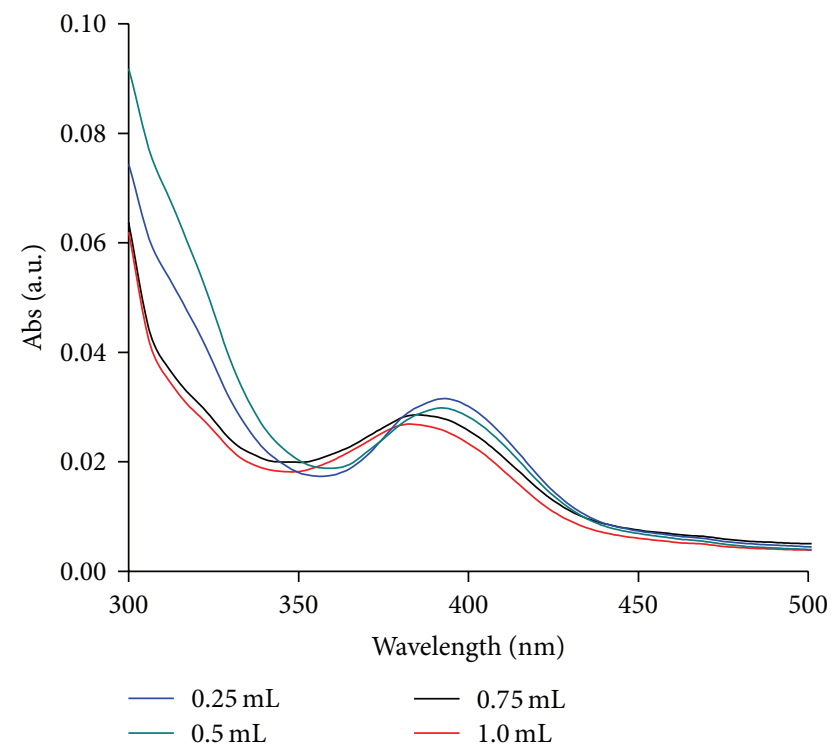

(b)

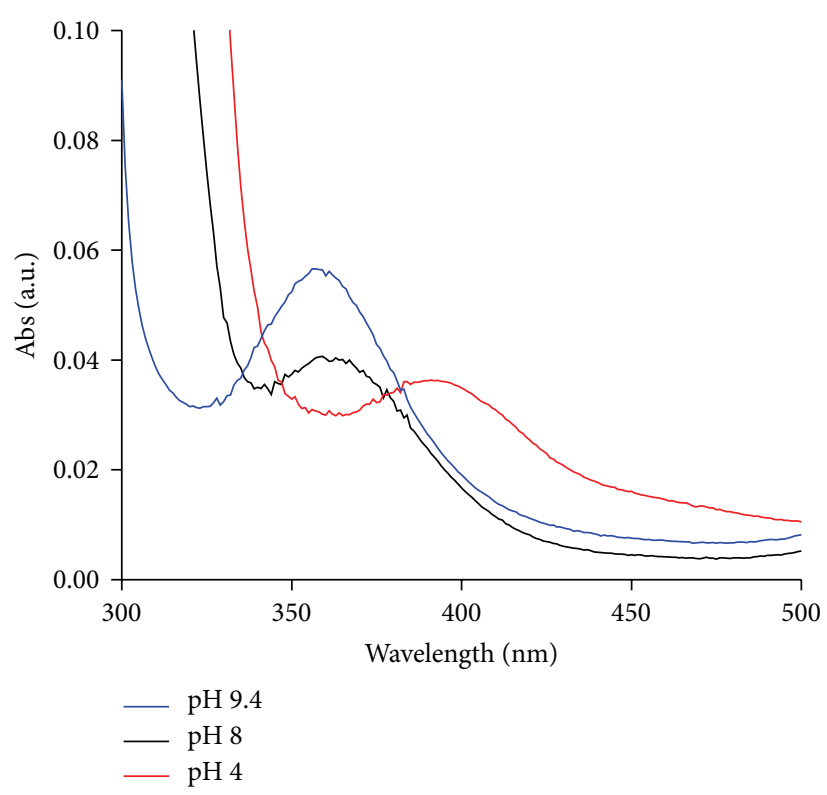

(c)

FIGURE 1: UV-Vis spectra recorded for (a) optimization of reducing agent (hydrazine monohydrate, $0.2 \mathrm{M}$ ), resulting in a blue shift in $\lambda_{\max }$ from $391 \mathrm{~nm}$ to $366 \mathrm{~nm}$ with increase in absorbance at various concentrations; (b) optimization of TX-100 (0.5 M) at various concentrations, resulting in a red shift in $\lambda_{\max }$ from $382 \mathrm{~nm}$ to $393 \mathrm{~nm}$ with increase in absorbance; and (c) (selected spectra) optimization of pH study where we observed a blue shift in $\lambda_{\max }$ from $391 \mathrm{~nm}$ to $357 \mathrm{~nm}$ with increase in absorbance. Further detail is given in the experimental section.

of such materials is mainly dependent on size and morphology [26]. The present study describes the synthesis of Ni NSs with controlled morphologies via a modified hydrazine reduction route using three typical $\mathrm{pH}$ values. SEM images show that Ni NSs samples prepared at $\mathrm{pH} 9.4$ consisted of mixed structures of hexagonal nanosheets with smooth surfaces and well-dispersed spherical nanoparticles with rough surfaces but uniform in size. To determine the dynamics of surfactant stabilized nanoparticle size and growth patterning at different $\mathrm{pH}$ values in the lyotropic liquid crystalline medium, SEM images were recorded; subsequent data are shown in Figure 4 and Figure S-1 to Figure S-2 of the supplementary data available online at http://dx.doi.org/10.1155/2014/126103.

These data demonstrate that mixed structures of fine nickel nanosheets (Ni NSs) and rough-surfaced nickel nanoparticles (Ni NPs) are clearly seen at $\mathrm{pH}$ 9.4. The method is introduced for synthesis of mixed nanostructures 


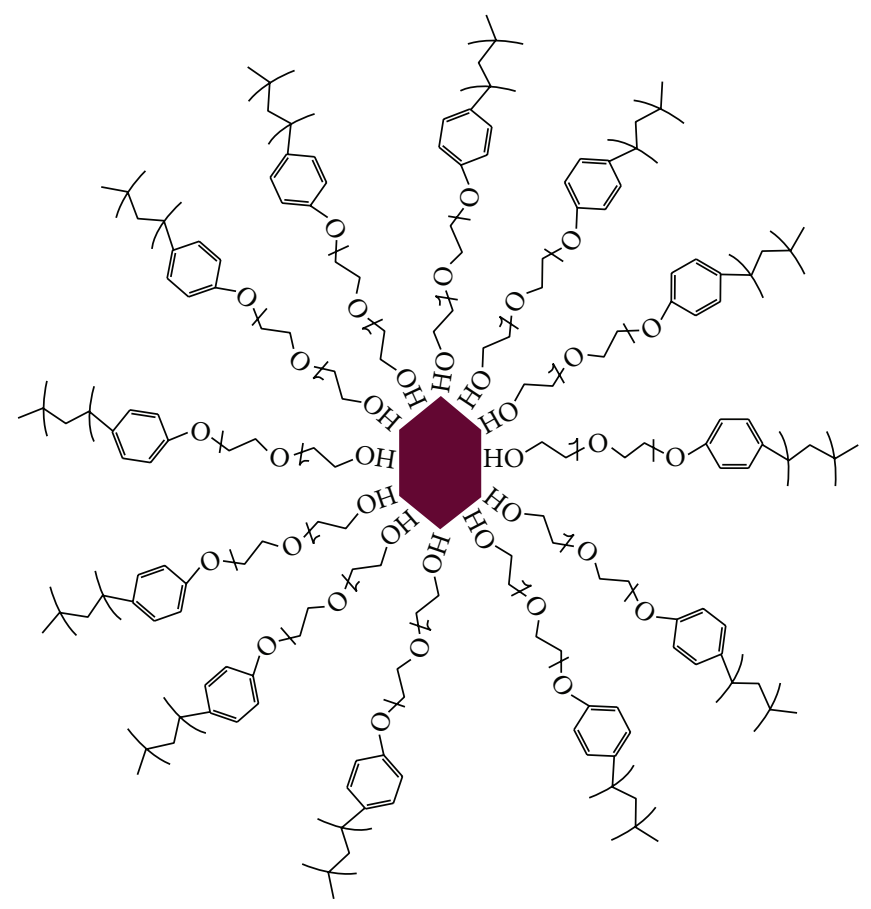

FIGURE 2: Schematic representation of the TX-100 stabilized Ni NSs.

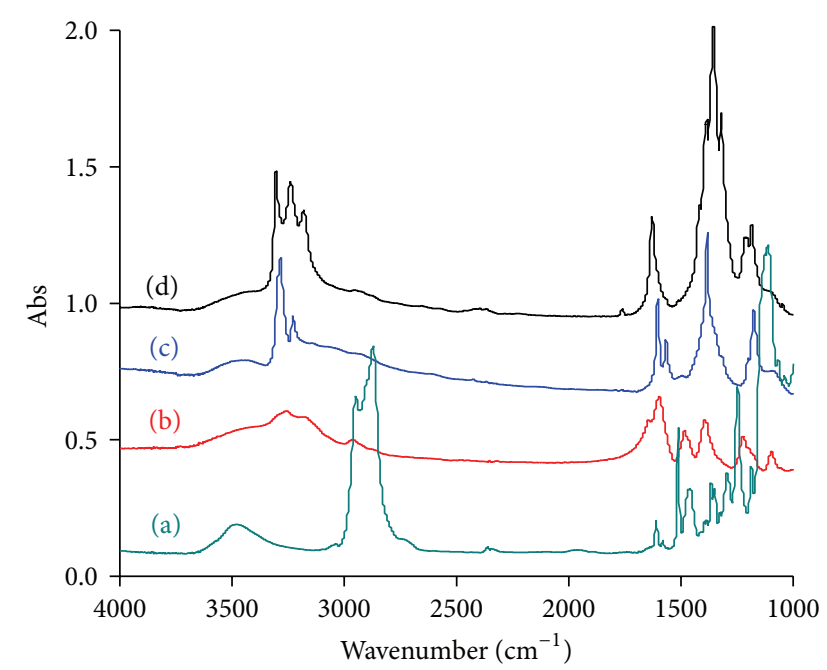

FIGURE 3: FTIR spectra of (a) standard TX-100 solution recorded on ATR; (b) TX-100 stabilized Ni NSs at pH 9.4; (c) TX-100 stabilized Ni NSs at pH 7.3; and (d) TX-100 stabilized Ni NSs at pH 4.2.

containing highly ordered assemblies of 2D nanosheets/foils with smooth surfaces and thicknesses in the range of 24$240 \mathrm{~nm}$, while the mean thickness is $72 \mathrm{~nm}$; these nanosheets are found to be $0.9-7.0 \mu \mathrm{m}$ across, averaging $3.1 \mu \mathrm{m}$, and the size of spherical nickel nanoparticles ranged from 8 to $300 \mathrm{~nm}$, with the observed average size of $45 \mathrm{~nm}$ in $\mathrm{pH} 9.4$.
These Ni NSs were used as heterogeneous catalysts for the reduction/degradation of a number of organic dyes and found to be highly active catalysts, recoverable even after several uses. Such nanostructures may also be employed for the development of innovative devices promising unique properties in the fields of microelectronics and sensors.

3.1.4. X-Ray Diffraction (XRD) Study of TX-100 Stabilized Ni NSs. The phase compositions of crystal structures of these products were analyzed by X-ray diffraction. Figure 5 shows the XRD pattern of powdered Ni NSs which corresponds to the formation of nanosheets/foils.

For the Ni NSs obtained by the modified hydrazine reduction route and separation by solvent evaporation at normal temperature (noncentrifuged sample), a $\mathrm{Ni}(\mathrm{OH})_{2}$ layer on the nanoparticle was predictable from the XRD results of sample 1 (Figure 5). The XRD peaks (at $2 \theta$ values of $22.01^{\circ}, 30.72^{\circ}$, and $\left.33.11^{\circ}\right)$ corresponding to the Miller indices (001), (100), and (101), respectively, are consistent with those reported elsewhere and attributed to formation of $\mathrm{Ni}(\mathrm{OH})_{2}$ $[27,28]$. The nanosized nickel showed only two characteristic peaks of pure face centered cubic ( $\mathrm{fcc}$ ) nickel (at $2 \theta$ values of $44.89^{\circ}$ and $53.44^{\circ}$ ), assigned to Miller indices (111) and (200), as depicted in Figure 4. Similar results have also been observed previously in the literature [29].

Further, it can be seen from the XRD results that all diffraction peaks of sample 2, collected by separating the nanosized particles from the aqueous medium by ultrahigh centrifugation (i.e., $22000 \mathrm{rpm}$ ), resulted from the formation of purely fcc phase nickel structures, illustrating the existence 


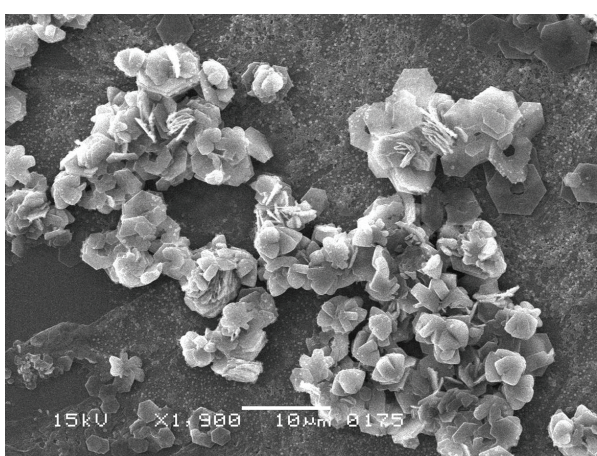

(a)

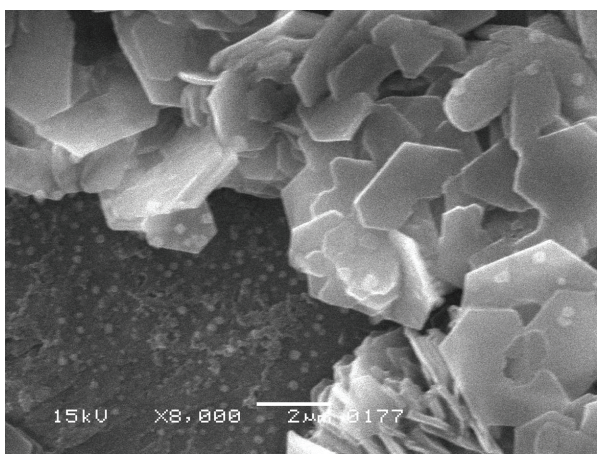

(c)

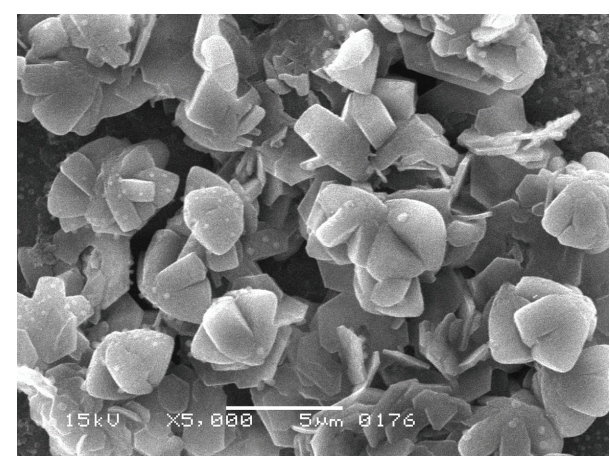

(b)

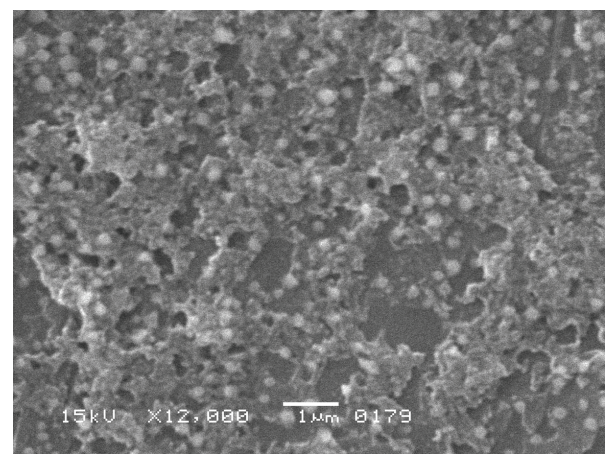

(d)

FIgURE 4: SEM images of TX-100 stabilized Ni NSs obtained at pH 9.4.

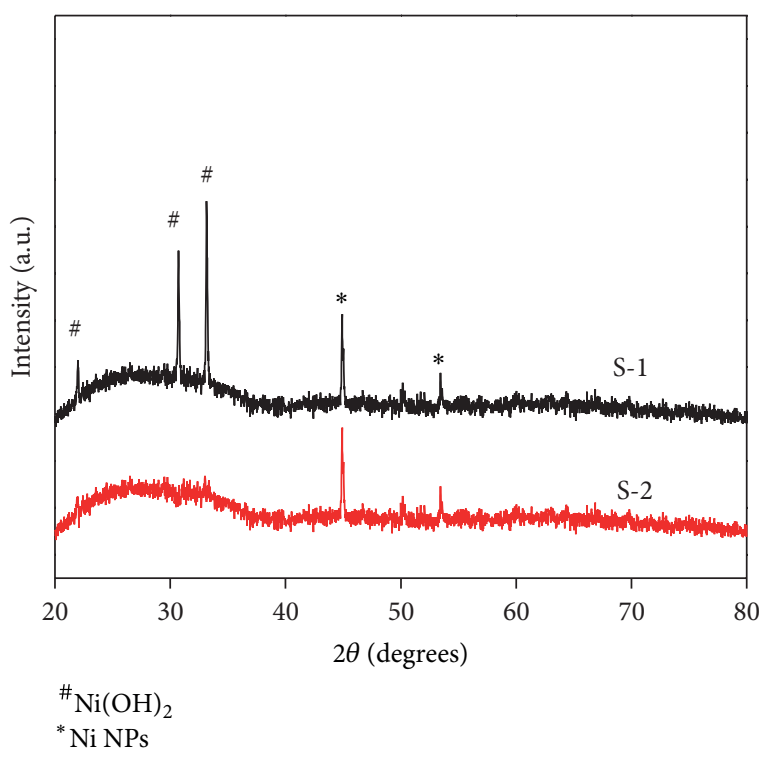

FIGURE 5: XRD patterns of TX-100 stabilized Ni NSs obtained by modified hydrazine reduction route, sample (1) noncentrifuged products and sample (2) centrifuged products of the as-prepared nanosized nickel structures.

of only metallic nickel particles [30]. We obtained mixed crystal structures with typical diffraction patterns indicating the formation of fcc phase Ni NPs for Ni-based nanosheets/foils similar to those proposed in the literature [31] and confirmed by surface morphology SEM images.

\section{Application of TX-100 Stabilized Ni NSs}

4.1. Reduction/Degradation of Dyes. Further experiments were performed for dye degradation/reduction with $\mathrm{NaBH}_{4}$ in both the presence and absence of nanoscale nickel catalysts. To investigate the catalytic activity of Ni NSs, the rates of degradation for several organic dyes, such as rose bengal (RB), eosin-b (EB), eriochrome black-t (ECBT), methylene blue (MB), and mixture of all four dyes, were measured using UV-Vis irradiation. The results obtained from these investigations are presented in Figures 6(a)-6(e) and the corresponding initial results for an un-catalyzed reactions to access the capability of reducing agent along with dyes in a similar sample environment are provided as supporting data, Figures S3(a)-S3(e).

Dyes were chosen because they produce different color shades in degraded and undegraded forms. The study showed that newly fabricated Ni NSs were excellent catalysts. Progress of the degradation of each dye was followed by the decrease in absorbance at their respective $\lambda_{\max }$ values in the UV-Vis spectral range; similar studies have been reported previously for the reduction of an organic dye [32]. That study showed reduction of acridine orange in $30 \mathrm{~min}$ using Pd nanoparticles via a homogeneous catalytic approach [32]. In contrast, $\mathrm{Ni}$ NSs were used as catalysts following a heterogeneous 

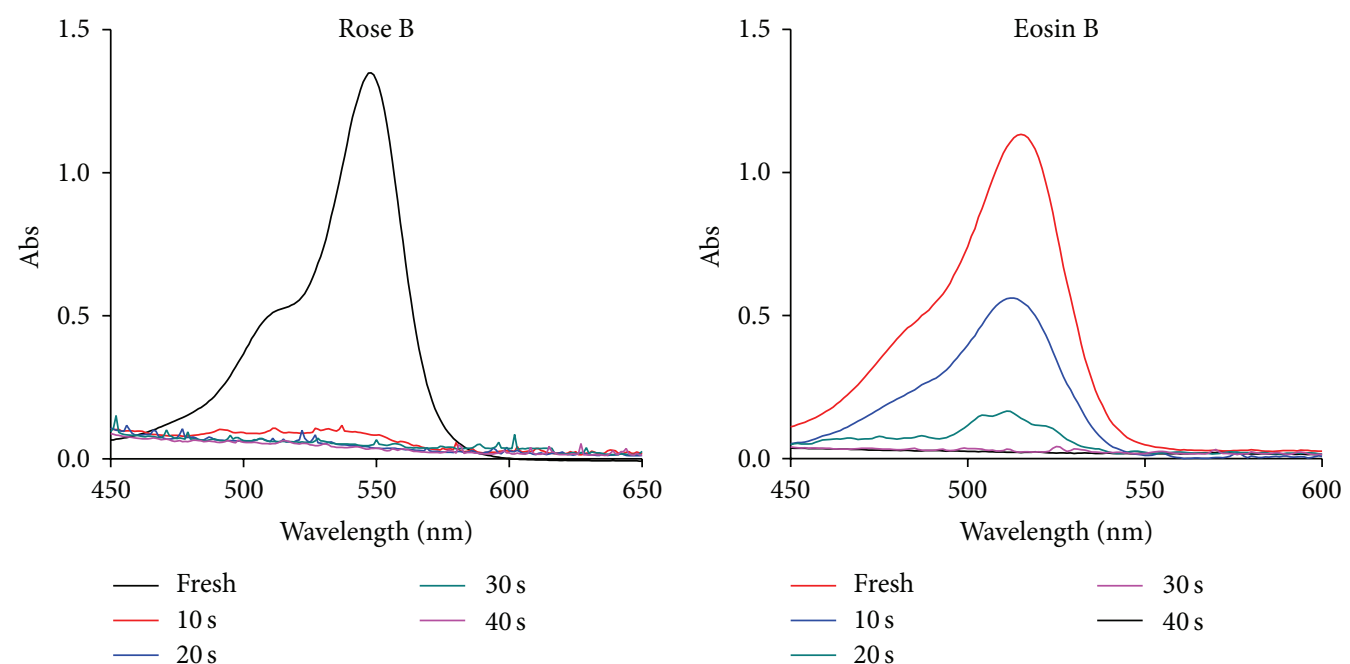

(a)
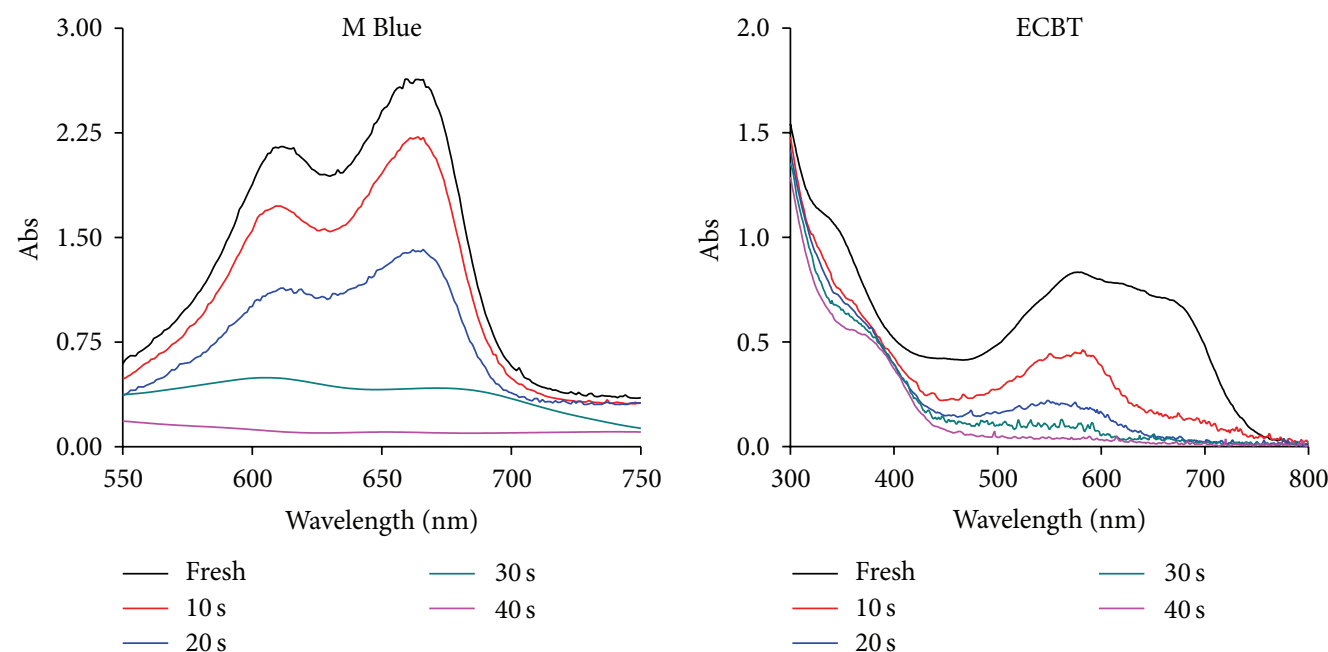

(c)

(d)

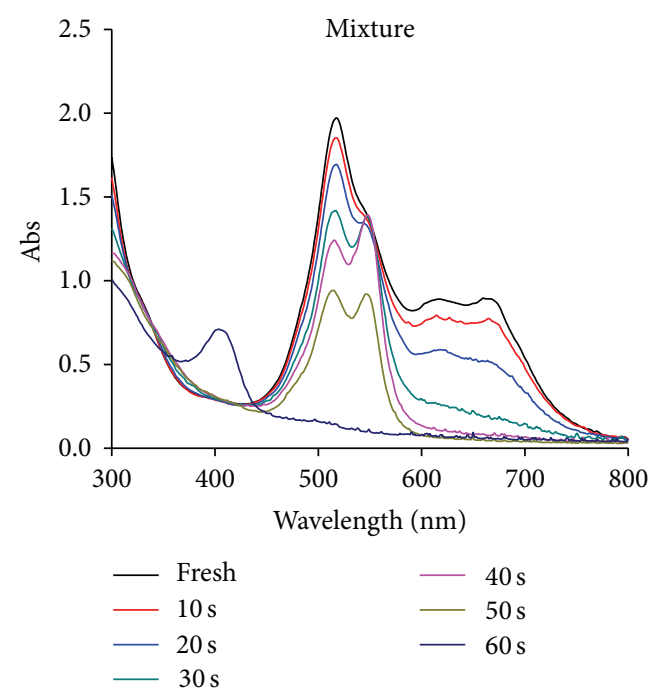

(e)

FiguRE 6: UV-Vis spectral analysis for catalytic reduction/degradation of a variety of dyes: (a) $0.02 \mathrm{mM} \mathrm{RB}$; (b) $0.02 \mathrm{mM}$ EB; (c) $0.02 \mathrm{mM}$ MB; (d) $0.02 \mathrm{mM} \mathrm{ECBT;} \mathrm{and} \mathrm{(e)} \mathrm{mixture} \mathrm{of} \mathrm{all} \mathrm{four} \mathrm{dyes,} \mathrm{carried} \mathrm{out} \mathrm{in} 4.0 \mathrm{~mL}$ of deionized water with $0.01 \mathrm{mM} \mathrm{NaBH}_{4}$ in the presence of a fixed amount of TX-100 stabilized Ni NSs $(0.2 \mathrm{mg})$ obtained at pH 9.4. 
TABLE 1: Catalytic degradation of organic dyes using Ni NPs as catalyst.

\begin{tabular}{|c|c|c|c|c|c|c|}
\hline Dye & Substrate & Time (s) & Rate $\left(k_{1}\right)$ & $\%$ Yield & TON & TOF \\
\hline $\mathrm{RB}$ & Ni NPs & 40 & 0.0549 & 96.122 & 37334.93 & 1.59 \\
\hline $\mathrm{EB}$ & Ni NPs & 40 & 0.1143 & 98.045 & 5669.89 & 3.31 \\
\hline ECBT & Ni NPs & 40 & 0.0814 & 95.558 & 6417.47 & 2.36 \\
\hline MB & Ni NPs & 40 & 0.0382 & 97.331 & 4328.65 & 1.11 \\
\hline Mixture & Ni NPs & 60 & 0.0139 & 95.504 & - & 4.03 \\
\hline
\end{tabular}

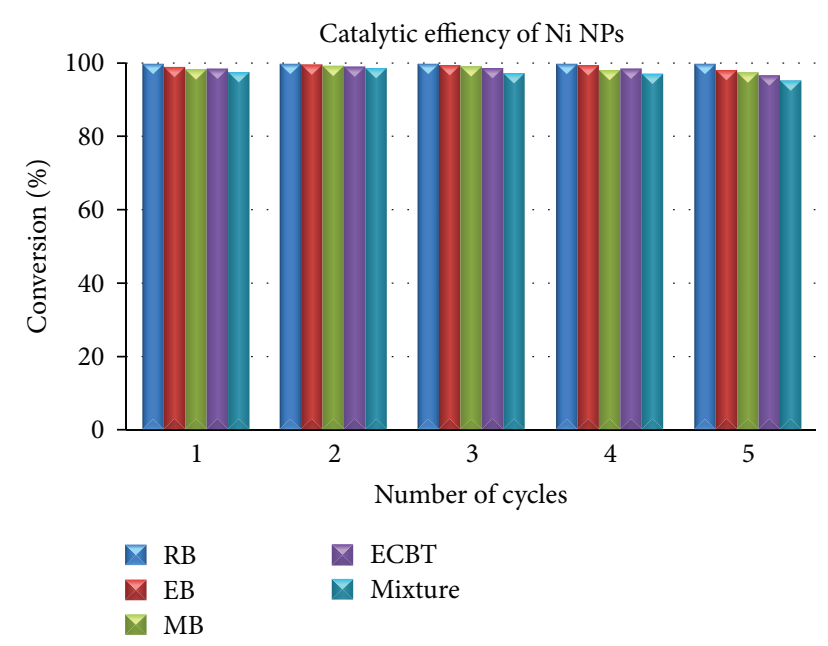

FIGURE 7: Histogram showing the recovery of TX-100 stabilized Ni NSs/NPs immobilized on a glass surface (the same catalysts) and used for five cycles that still resulted in fast reduction of the dyes (i.e., RB, EB, MB, ECBT, and mixture) with high yields.

catalytic approach which is much more safe, cost effective, and advantageous, allowing their use several times with good efficiency. The present study revealed that RB and EB dyes were not reduced without the use of catalyst, whereas $M B$ and ECBT showed little decrease in absorbance with time. However, in the presence of catalysts, very fast degradation (i.e., 20-60 s) of all used dyes was observed.

The efficiency of TX-100 stabilized Ni NSs/NPs for reduction/degradation of the dyes (i.e., RB, EB, MB, and ECBT) was monitored. Figure 7 demonstrates how Ni NPs immobilized on a glass surface (the same catalysts) and used for five cycles still resulted in fast reduction of the dyes (i.e., RB, EB, MB, and ECBT) with high yields; reaction progress was monitored by UV-Vis spectroscopy.

In addition it was also observed that newly fabricated TX100 stabilized Ni NSs catalyzed dye reduction to nearly $100 \%$ in the presence of $\mathrm{NaBH}_{4}$ in a very short time, that is, 20$60 \mathrm{~s}$; furthermore, the same catalysts could be recovered and reused several times.

4.2. Catalyst Efficiency. The catalyst efficiency for degradation of organic dyes was calculated by employing the equations for measuring extent of degradation, turnover number (TON), and mean turnover frequency (TOF) $[33,34]$.
The dye degradation percentage ratio was calculated using the following formula:

$$
\text { Degradation }(\%)=\left[\frac{\mathrm{Abs}_{0}-\mathrm{Abs}_{t}}{\mathrm{Abs}_{0}}\right] \times 100 .
$$

$\left(\mathrm{Abs}_{0}\right)$ is the initial absorbance of dye and $\left(\mathrm{Abs}_{t}\right)$ is the absorbance of dye on time $t$. The turnover number (TON), describing the ratio of moles of product obtained to the moles of catalyst used, was calculated using the following formula:

$$
\mathrm{TON}=\left(\frac{\text { moles dye converted }}{\text { mole of catalyst }}\right) \times 100 .
$$

The cumulative TON for dyes at given times (i.e., for maximum degradation) to estimate the efficiency of catalysts is presented in Table 1.

The mean turnover frequency (TOF) was also calculated, that is, the rate of change of TON for a reaction in any given recycling sequence (reflecting reaction rate for a fixed catalyst loading), and was determined as the ratio of cumulative TON/time from formula (4); the results obtained are also given in Table 1.

Consider

$$
\mathrm{TOF}=\frac{-k_{1}\left(s^{-1}\right) \times[\text { Analyte }]_{t=0}(M)}{[\mathrm{Ni} \mathrm{NPs}](M)} .
$$

From the above illustrations and the calculated values for TON and TOF, it can be seen that Ni NPs possess highly active surfaces to degrade large number of molecules of each dye per unit amount of the catalyst in a very short time. These Ni NPs once immobilized onto the glass surface can be used many times to get good yields. Hence, it is observed that the Ni NSs synthesized by a simple and inexpensive protocol showed good catalytic efficiency for the degradation of both individual and mixed organic dyes and thus applicability for the removal of pollutants from water systems.

\section{Conclusions}

The new simple and cost effective method is introduced for synthesis of mixed nanoscale nickel structures containing highly ordered assemblies of 2D nanosheets with absolutely smooth surfaces and sheet thicknesses in the range of 24$240 \mathrm{~nm}$ (average thickness is $72 \mathrm{~nm}$ ); these nanosheets are found to be $0.9-7.0 \mu \mathrm{m}$ across, averaging $3.1 \mu \mathrm{m}$, and the size of spherical nickel nanoparticles is in the range of $8-300 \mathrm{~nm}$, with the observed average size being $45 \mathrm{~nm}$. The extended 
complexity and functionality of the nanoscale systems are predictable from efficient linkages employed by the $\mathrm{OH}$ group of TX-100 molecules and Ni particles in a lyotropic liquid crystalline medium. These NSs were used for the reduction of organic dyes and found to be highly active and efficient catalysts.

\section{Conflict of Interests}

The authors declare that there is no conflict of interests regarding the publication of this paper.

\section{Acknowledgments}

The authors acknowledge the Higher Education Commission, Islamabad, Pakistan, for funding and the National Centre of Excellence in Analytical Chemistry, University of Sindh, Jamhoro, Pakistan, and the Interface Analysis Centre, School of Physics, University of Bristol, Bristol, United Kingdom, for provision of laboratory facilities during this research. Special thanks go to Dr. Sean Davis and Dr. Loren Picco of the University of Bristol, Bristol, United Kingdom, for their kind assistance with microscopy studies.

\section{References}

[1] M. A. Breimer, G. Yevgeny, S. Sy, and O. A. Sadik, "Incorporation of metal nanoparticles in photopolymerized organic conducting polymers: a mechanistic insight," Nano Letters, vol. 1, no. 6, pp. 305-308, 2001.

[2] H. He, S. Yang, K. Yu, Y. Ju, C. Sun, and L. Wang, "Microwave induced catalytic degradation of crystal violet in nano-nickel dioxide suspensions," Journal of Hazardous Materials, vol. 173, no. 1-3, pp. 393-400, 2010.

[3] R. Andreozzi, V. Caprio, A. Insola, and R. Marotta, "Advanced Oxidation Processes (AOP) for water purification and recovery," Catalysis Today, vol. 53, no. 1, pp. 51-59, 1999.

[4] J. M. Hermann, "Heterogeneous photocatalysis: fundamentals and applications to the removal of various types of aqueous pollutants," Catalysis Today, vol. 53, no. 1, pp. 115-129, 1999.

[5] M. H. Liao, K. Y. Wu, and D. H. Chen, "Fast adsorption of crystal violet on polyacrylic acid-bound magnetic nanoparticles," Separation Science and Technology, vol. 39, no. 7, pp. 1563-1575, 2005.

[6] O. Gezici, M. Küçükosmanoǧlu, and A. Ayar, "The adsorption behavior of crystal violet in functionalized sporopolleninmediated column arrangements," Journal of Colloid and Interface Science, vol. 304, no. 2, pp. 307-316, 2006.

[7] J. J. Jones and J. O. Falkinham III, "Decolorization of malachite green and crystal violet by waterborne pathogenic mycobacteria," Antimicrobial Agents and Chemotherapy, vol. 47, no. 7, pp. 2323-2326, 2003.

[8] F. A. Alshamsi, A. S. Albadwawi, M. M. Alnuaimi, M. A. Rauf, and S. S. Ashraf, "Comparative efficiencies of the degradation of Crystal Violet using UV/hydrogen peroxide and Fenton's reagent," Dyes and Pigments, vol. 74, no. 2, pp. 283-287, 2007.

[9] M. Saquib and M. Muneer, " $\mathrm{TiO}_{2}$-mediated photocatalytic degradation of a triphenylmethane dye (gentian violet), in aqueous suspensions," Dyes and Pigments, vol. 56, no. 1, pp. 3749, 2003.
[10] J. Hong, C. Sun, S.-G. Yang, and Y.-Z. Liu, "Photocatalytic degradation of methylene blue in $\mathrm{TiO}_{2}$ aqueous suspensions using microwave powered electrodeless discharge lamps," Journal of Hazardous Materials, vol. 133, no. 1-3, pp. 162-166, 2006.

[11] Z. He, C. Sun, S. Yang, Y. Ding, H. He, and Z. Wang, "Photocatalytic degradation of rhodamine $\mathrm{B}$ by $\mathrm{Bi}_{2} \mathrm{WO}_{6}$ with electron accepting agent under microwave irradiation: mechanism and pathway," Journal of Hazardous Materials, vol. 162, no. 2-3, pp. 1477-1486, 2009.

[12] Y. M. Ju, S. G. Yang, Y. C. Ding, C. Sun, A. Q. Zhang, and L. H. Wang, "Microwave-assisted rapid photocatalytic degradation of malachite green in $\mathrm{TiO}_{2}$ suspensions: mechanism and pathways," Journal of Physical Chemistry A, vol. 11, no. 44, pp. 11172-11177, 2008.

[13] C. C. Chen, H. J. Liao, C. Y. Cheng, C. Y. Yen, and Y. C. Chung, "Biodegradation of crystal violet by Pseudomonas putida," Biotechnology Letters, vol. 29, no. 3, pp. 391-396, 2007.

[14] K. Banerjee, P. N. Cheremisinoff, and S. L. Cheng, "Sorption of organic contaminants by fly ash in a single solute system," Environmental Science \& Technology, vol. 29, no. 9, pp. 22432251, 1995.

[15] R. Comparelli, E. Fanizza, M. L. Curri, P. D. Cozzoli, G. Mascolo, and A. Agostiano, "UV-induced photocatalytic degradation of azo dyes by organic-capped $\mathrm{ZnO}$ nanocrystals immobilized onto substrates," Applied Catalysis B, vol. 60, no. 1-2, pp. 1-11, 2005.

[16] M. Vaez, A. Z. Moghaddam, N. M. Mahmoodi, and S. Alijani, "Decolorization and degradation of acid dye with immobilized titania nanoparticles," Process Safety and Environmental Protection, vol. 90, no. 1, pp. 56-64, 2012.

[17] P. K. Rastogi, V. Ganesan, and S. Krishnamoorthi, "Microwave assisted polymer stabilized synthesis of silver nanoparticles and its application in the degradation of environmental pollutants," Materials Science and Engineering B, vol. 77, no. 6, pp. 456-461, 2012.

[18] B. Sahoo, S. K. Sahu, S. Nayak, D. Dhara, and P. Pramanik, "Fabrication of magnetic mesoporous manganese ferrite nanocomposites as efficient catalyst for degradation of dye pollutants," Catalysis Science \& Technology, vol. 2, no. 7, pp. 1367-1374, 2012.

[19] Z. A. Tagar, Sirajuddin, N. Memon et al., "Facile synthesis, characterization and catalytic function of gelatin stabilized gold nanoparticles," Pakistan Journal of Analytical and Environmental Chemistry, vol. 13, no. 1, p. 70, 2012.

[20] N. H. Kalwar, Sirajuddin, K. R. Hallam et al., "Fabrication of small 1-threonine capped nickel nanoparticles and their catalytic application," Applied Catalysis A, vol. 453, pp. 54-59, 2013.

[21] N. H. Kalwar, Sirajuddin, S. T. H. Sherazi et al., "Synthesis of 1-methionine stabilized nickel nanowires and their application for catalytic oxidative transfer hydrogenation of isopropanol," Applied Catalysis A, vol. 400, no. 1-2, pp. 215-220, 2011.

[22] K. Nouneh, M. Oyama, R. Diaz, M. Abd-Lefdil, I. V. Kityk, and M. Bousmina, "Nanoscale synthesis and optical features of metallic nickel nanoparticles by wet chemical approaches," Journal of Alloys and Compounds, vol. 509, no. 19, pp. 58825886, 2011.

[23] H. Amekura, Y. Takeda, and N. Kishimoto, "Criteria for surface plasmon resonance energy of metal nanoparticles in silica glass," Nuclear Instruments and Methods in Physics Research B, vol. 222, no. 1-2, pp. 96-104, 2004.

[24] D. Liu, S. Ren, H. Wu, Q. Zhang, and L. Wen J, “Morphology control in synthesis of nickel nanoparticles in the presence of 
polyvinylpyrrolidone (PVPK30)," Journal of Materials Science, vol. 43, no. 6, pp. 1974-1978, 2008.

[25] P. S. Denkova, L. van Lokeren, I. Verbruggen, and R. Willem, "Self-aggregation and supramolecular structure investigations of triton X-100 and SDP2S by NOESY and diffusion ordered NMR spectroscopy," Journal of Physical Chemistry B, vol. 112, no. 35, pp. 10935-10941, 2008.

[26] L. Qi, "Colloidal chemical approaches to inorganic micro- and nanostructures with controlled morphologies and patterns," Coordination Chemistry Reviews, vol. 254, no. 9-10, pp. 10541071, 2010.

[27] R. Chen, Q. Wang, Y. Du, W. Xing, and N. Xu, "Effect of initial solution apparent $\mathrm{pH}$ on nano-sized nickel catalysts in $\mathrm{p}$ nitrophenol hydrogenation," Chemical Engineering Journal, vol. 145, no. 3, pp. 371-376, 2009.

[28] F. Tao, M. Guan, Y. Zhou, L. Zhang, Z. Xu, and J. Chen, "Fabrication of nickel hydroxide microtubes with micro- and nano-scale composite structure and improving electrochemical performance," Crystal Growth and Design, vol. 8, no. 7, pp. 21572162, 2008.

[29] Y. Du, H. Chen, R. Chen, and N. Xu, "Synthesis of $p$ aminophenol from $p$-nitrophenol over nano-sized nickel catalysts," Applied Catalysis A, vol. 277, no. 1-2, pp. 259-264, 2004.

[30] L. Bai, F. Yuan, and Q. Tang, "Synthesis of nickel nanoparticles with uniform size via a modified hydrazine reduction route," Materials Letters, vol. 62, no. 15, pp. 2267-2270, 2008.

[31] B. Zhang, X. Ye, W. Dai, W. Hou, and Y. Xie, "Biomoleculeassisted synthesis and electrochemical hydrogen storage of porous spongelike $\mathrm{Ni}_{3} \mathrm{~S}_{2}$ nanostructures grown directly on nickel foils," Chemistry, vol. 12, no. 8, pp. 2337-2342, 2006.

[32] S. Nath, S. Praharaj, S. Panigrahi, S. Basu, and T. Pal, "Photochemical evolution of palladium nanoparticles in Triton X100 and its application as catalyst for degradation of acridine orange," Current Science, vol. 92, no. 6, pp. 786-790, 2007.

[33] M. C. Rosu, R. C. Suciu, I. Kasco, S. V. Dreve, E. Indrea, and T. D. Silipas, "A spectroscopic study of dyes decomposition by irradiated nanocrystalline $\mathrm{TiO}_{2}$," Journal of Physics: Conference Series, vol. 182, no. 1, Article ID 012078, 2009.

[34] Y. Zhang and T. Ren, "Silica supported ruthenium oxide nanoparticulates as efficient catalysts for water oxidation," Chemical Communications, vol. 48, no. 89, pp. 11005-11007, 2012. 

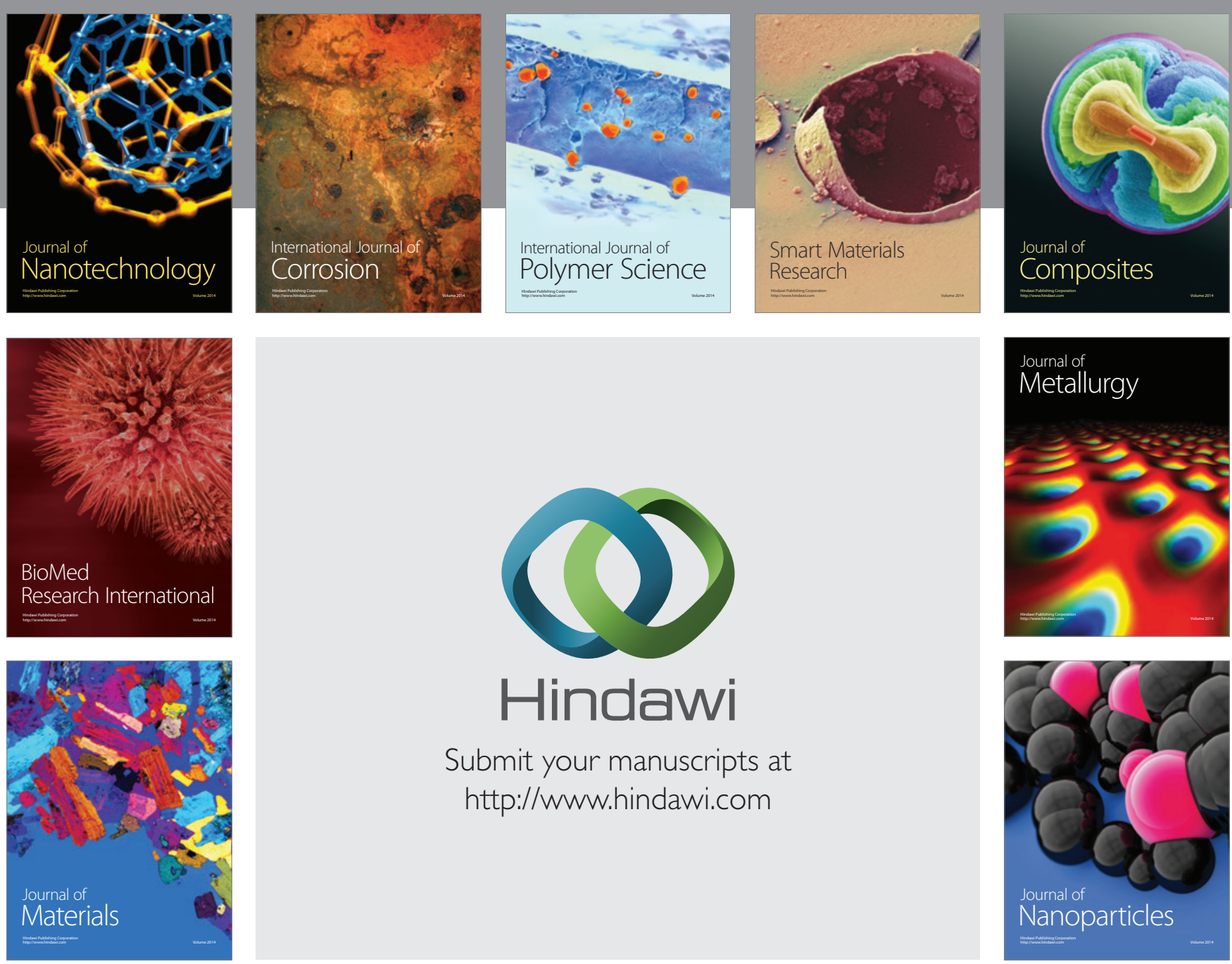

Submit your manuscripts at http://www.hindawi.com
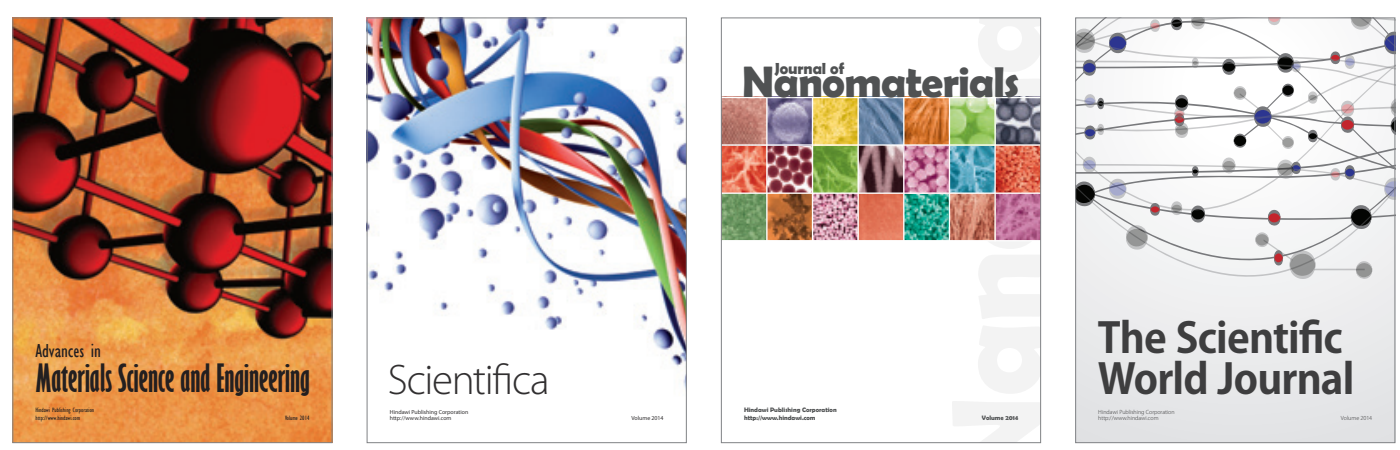

\section{The Scientific World Journal}
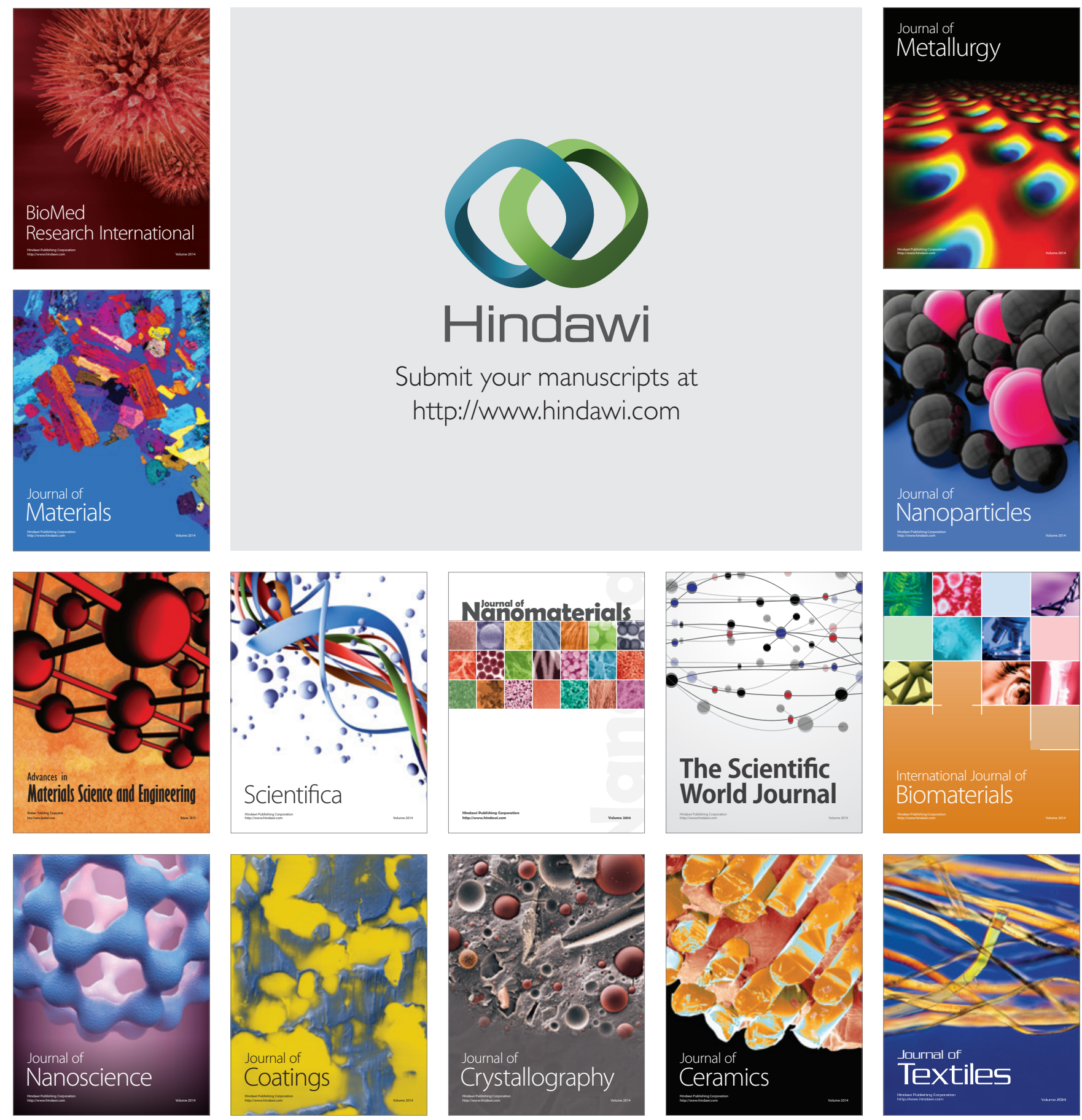